\title{
Familial predisposition of myeloid malignancies: biological and clinical significance of recurrent germ line mutations
}

\author{
Hirotaka Matsui ${ }^{1}$ (D)
}

Received: 12 June 2017 / Accepted: 14 June 2017 / Published online: 19 June 2017

(C) The Japanese Society of Hematology 2017

\section{Introduction}

The World Health Organization classification of myeloid neoplasms and acute leukemias was updated in 2016 from its previous 2008 version. In the 2016 version, enormous progress made in the field of genetic analysis, brought about by recent advances in the comprehensive identification of gene mutations, was adopted throughout the classification [1]. One such example is the addition of a section describing germ line mutations that predispose patients to myeloid neoplasms (Table 1). This section classifies the entity into three categories as follows: (1) myeloid neoplasms with a germ line predisposition and without a preexisting disorder or organ dysfunction, (2) those in patients with preexisting platelet disorders, and (3) those in patients with other organ dysfunctions. Although most hematological malignancies occur based on accumulating somatic mutations, we should always be aware of a familial predisposition for hematological malignancies, and thus carefully probe patients on whether they have a family history of similar hematological disorders and/or other malignancies. Indeed, case reports exist describing the development of hematological malignancies originating from donor-derived cells harboring germ line gene mutations after allogeneic hematopoietic stem cell transplantation. In these cases, intrinsic and/or extrinsic stresses to hematopoietic stem/progenitor cells during the reconstitution of bone marrow may trigger the transformation of normal cells into

Hirotaka Matsui

hmatsui@kumamoto-u.ac.jp

1 Department of Molecular Laboratory Medicine, Graduate School of Medical Sciences, Kumamoto University, 1-1-1 Honjo, Chuo-ku, Kumamoto 860-8556, Japan malignant clones. In this series of review articles, a total of four specialists, who have made remarkable contributions to the field of familial hematological malignancies, present a recent understanding of these syndromes. Herein, I will briefly summarize the essence of these review articles.

\section{Familial platelet disorder with predisposition to acute myelogenous leukemia}

The first gene shown to be involved in this category of the disease is $R U N X 1$, where germ line deletions, or nonsense, splice site or missense mutations have been identified mainly in the runt-homology domain, which plays a role in sequence-specific DNA binding. These germ line mutations cause impaired megakaryopoiesis that gives rise to inherited thrombocytopenia and subsequent acute myeloid leukemia (AML). This syndrome, referred to as familial platelet disorder (FPD)/AML [2], leads to the development of myeloid neoplasms in patients, typically when in their $30 \mathrm{~s}$ or $40 \mathrm{~s}$. In this disorder, somatic mutations of TET2, $A S X L 1, I D H 1 / 2$ or $C D C 25 C$ genes, in addition to the germ line RUNX1 mutation, are supposed to contribute to the malignant transformation of hematopoietic cells. It has also been shown that $20-60 \%$ of individuals in affected families develop AML. On this issue, Drs. Hayashi and Harada review recent progress in the understanding of this disorder [3].

\section{Germ line ETV6 mutations}

Recently, germ line variants of the ETV6 gene have also been shown to cause familial thrombocytopenia that frequently progresses into diverse hematologic malignancies, 
Table 1 Classification of myeloid neoplasms with germ line predisposition (WHO 2016)

Myeloid neoplasms with a germ line predisposition and without a
preexisting disorder or organ dysfunction
AML with germ line CEBPA mutation
Myeloid neoplasms with germ line DDX41 mutation
Myeloid neoplasms with germ line predisposition and preexisting
platelet disorders
Myeloid neoplasms with germ line RUNX1 mutation
Myeloid neoplasms with germ line ANKRD26 mutation
Myeloid neoplasms with germ line ETV6 mutation
Myeloid neoplasms with germ line predisposition and other organ
dysfunction
Myeloid neoplasms with germ line GATA2 mutation
Myeloid neoplasms associated with BM failure syndrome
Myeloid neoplasms associated with telomere biology disorders
JMML associated with neurofibromatosis, Noonan syndrome or
Noonan syndrome-like disorders
Myeloid neoplasms associated with Down syndrome

$B M$ bone marrow, $J M M L$ juvenile myelomonocytic leukemia

including $\mathrm{B}$ acute lymphoblastic leukemia, mixed-lineage leukemia and myelodysplastic syndrome (MDS) [4, 5]. This gene encodes an important transcriptional repressor that possesses a highly conserved ETS DNA-binding domain; most germ line mutations reported so far are found within this domain or within a linker inhibitory domain that mediates the binding of the protein to DNA. Physiologically, the ETV6 transcription factor is involved in early embryonic development and adult hematopoiesis as well as in erythroid and megakaryocytic differentiation and maturation. Accumulating evidence reveals that some ETV6 mutants (p.P214L, p.R369Q and p.R399C and the others) identified from affected families completely or at least partially lose their nuclear localization property, thus causing the impaired function of the protein as a transcriptional repressor. Although not extensively reviewed in this issue because of space limitations, germ line ANKRD26 mutations, in addition to ETV6 and RUNX1 mutations, have been shown to play a role in the development of myeloid malignancies accompanied by thrombocytopenia [6].

\section{Germ line DDX41 mutations}

The $D D X 41$ germ line mutation was first described by Maciejewski's group in 2015 [7]. Hematological malignancies associated with the mutation are not usually accompanied by thrombocytopenia; the mutation is currently estimated to be involved in nearly $1-2 \%$ of inherited hematological malignancies. Of note, patients with DDX41 mutations develop AML and MDS at relatively advanced age, with a median age at diagnosis of 65 years, which is similar to sporadic cases. However, a recent paper reports pedigrees developing in lower onset of hematological malignancies [8]. Biologically, this gene encodes a DEAD-box type RNA helicase that have been shown to play various roles, including in pre-mRNA splicing and ribosome biogenesis. We recently reported that a $D D X 41$ p.R525H mutation, which is typically found as a somatic mutation in myeloid neoplasms, affects ribosomal biogenesis through the inhibition of pre-ribosomal RNA processing and that the expression of the mutant causes a certain ribosomopathy phenotype in hematopoietic cells that leads to a slower growth rate of cells [9]. DDX41 is also likely to take part in innate immune regulation. However, the mechanisms by which the germ line, as well as the somatic $D D X 41$ mutation, causes hematological disorders are still unclear. Therefore, further basic experiments and analysis of a large cohort of affected families are clearly warranted to understand the roles of the mutation and to the establish treatment strategies. Dr. Cheah comprehensively reviews a recent understanding of $D D X 41$ in this issue [10].

\section{Germ line GATA2 mutations}

GATA2 deficiency, reviewed by Dr. Hirabayashi et al. in this issue, is a series of diseases caused by a germ line GATA2 gene mutation, which was first described in 2011 as a discrete syndrome $[11,12]$. The syndrome is characterized by congenital lymphedema, immunodeficiency involving a severe decrease in monocytes, B lymphocytes and NK cells that cause mycobacterial infection, and an earlier onset of MDS development. Since the GATA2 mutation is usually heterozygous, and the mutational position of the gene is spread over entire exons, and more than half the variants are a truncating type mutation, including frameshift, stop gain or splice site mutations, it is hypothesized that the syndrome is caused by the haploinsufficiency of the GATA2 protein. GATA2 is a transcription factor expressed in hematopoietic progenitors that is indispensable in hematopoietic cell differentiation. Although currently it is not completely known how a GATA2 mutation is involved in malignant transformation, it has recently been revealed that MDS with a germ line GATA2 mutation is strongly associated with monosomy 7 [13].

\section{Compliance with ethical standards}

Conflict of interest The author declares that he has no conflict of interest. 


\section{References}

1. Arber DA, Orazi A, Hasserjian R, Thiele J, Borowitz MJ, Le Beau MM, et al. The 2016 revision to the World Health Organization classification of myeloid neoplasms and acute leukemia. Blood. 2016;127(20):2391-405.

2. Song WJ, Sullivan MG, Legare RD, Hutchings S, Tan X, Kufrin D, et al. Haploinsufficiency of CBFA2 causes familial thrombocytopenia with propensity to develop acute myelogenous leukaemia. Nat Genet. 1999;23(2):166-75.

3. Hayashi Y, Harada Y, Huang G, Harada H. Myeloid neoplasms with germ line RUNX1 mutation. Int J Hematol. 2017. doi:10.1007/s12185-017-2258-5.

4. Zhang MY, Churpek JE, Keel SB, Walsh T, Lee MK, Loeb KR, et al. Germline ETV6 mutations in familial thrombocytopenia and hematologic malignancy. Nat Genet. 2015;47(2):180-5.

5. Feurstein S, Godley LA. Germline ETV6 mutations and predisposition to hematological malignancies. Int J Hematol. 2017. doi:10.1007/s12185-017-2259-4.

6. Noris P, Favier R, Alessi MC, Geddis AE, Kunishima S, Heller PG, et al. ANKRD26-related thrombocytopenia and myeloid malignancies. Blood. 2013;122(11):1987-9.

7. Polprasert C, Schulze I, Sekeres MA, Makishima H, Przychodzen B, Hosono N, et al. Inherited and somatic defects in DDX41 in myeloid neoplasms. Cancer Cell. 2015;27(5):658-70.
8. Lewinsohn M, Brown AL, Weinel LM, Phung C, Rafidi G, Lee MK, et al. Novel germ line DDX41 mutations define families with a lower age of MDS/AML onset and lymphoid malignancies. Blood. 2016;127(8):1017-23.

9. Kadono M, Kanai A, Nagamachi A, Shinriki S, Kawata J, Iwato $\mathrm{K}$, et al. Biological implications of somatic DDX41 p. $\mathrm{R} 525 \mathrm{H}$ mutation in acute myeloid leukemia. Exp Hematol. 2016;44(8):745-54.

10. Cheah JJC, Hahn CN, Hiwase DK, Scott HS, Brown AL. Myeloid neoplasms with germline DDX41 mutation. Int J Hematol. 2017. doi:10.1007/s12185-017-2260-y.

11. Hsu AP, Sampaio EP, Khan J, Calvo KR, Lemieux JE, Patel SY, et al. Mutations in GATA2 are associated with the autosomal dominant and sporadic monocytopenia and mycobacterial infection (MonoMAC) syndrome. Blood. 2011;118(10):2653-5.

12. Hirabayashi Y, Wlodarski MW, Kozyra E, Niemeyer CM (2017) Heterogeneity of GATA2-related myeloid neoplasms. Int J Hematol.

13. Wlodarski MW, Hirabayashi S, Pastor V, Stary J, Hasle H, Masetti R, et al. Prevalence, clinical characteristics, and prognosis of GATA2-related myelodysplastic syndromes in children and adolescents. Blood. 2016;127(11):1387-97. 